\title{
The MUSE experiment
}

\section{Addressing the proton radius puzzle via elastic muon scattering}

\author{
E. J. Downie
}

The George Washington University, Institute of Nuclear Studies, 725 21st St. NW, Washington, DC 20052, USA

\begin{abstract}
The proton radius puzzle is the difference between the proton radius as measured with electron scattering and in the excitation spectrum of atomic hydrogen, and that measured with muonic hydrogen spectroscopy. The MUSE experiment seeks to resolve this puzzle by simultaneously measuring elastic electron and muon scattering on the proton, in both charge states, thereby providing new information to the puzzle. MUSE addresses issues of two-photon effects, lepton universality and, possibly, new physics, while providing simultaneous form factor, and therefore radius, measurements with both muons and electrons.
\end{abstract}

\section{Introduction}

The proton radius puzzle arose in 2010 when the CREMA collboration measured the radius of the proton using the Lamb Shift in muonic hydrogen. Their result was $4 \%$ lower than the accepted value of the proton radius which had been gleaned from years of excitation spectrum measurements in atomic hydrogen and elastic electron scattering on the proton [1].

The radius can be extracted by measuring the slope of the form factor as $Q^{2}$ approaches zero in scattering experiments. In spectroscopy measurements, such as those performed by the CREMA collaboration, the small pertubations to the energy levels of an atom caused by the finite size of the proton, are measured and, from these perturbations, the radius is extracted.

Since 2010, many suggestions have been made as to the exact cause of the proton radius puzzle, but, to date, none of them has been widely accepted as the solution to the problem. Instead, it is now generally believed that a possible solution to the problem will come through further experimental investigation. The CREMA collaboration have begun an extended measurement series on heavier nuclei, lower $Q^{2}$ electron scattering experiments are planned at JLab and Mainz, and the MUSE collaboration has been formed to address the missing piece in the puzzle: elastic muon scattering on the proton.

The MUSE Experiment will take place at the $\pi \mathrm{M} 1$ beamline of the Paul Scherrer Institute (PSI), in Villigen, Switzerland. The $\pi \mathrm{M} 1$ beamline provides a secondary beam, a mixture of pions, muons

\footnotetext{
${ }^{a}$ On behalf of the MUSE Collaboration. EJD is funded by NSF grant 1309130; e-mail: edownie@gwu .edu
} 
and electrons. The beam qualities: low flux and large emittance have led to a unique experimental design to provide a simultaneous measurement of elastic electron and muon scattering. We will measure with both beam charge states, leading not only to a comparison of the radius as extracted simultaneously from electron and muon scattering, but also a check on the contribution of two-photon-effects, which change sign with the lepton charge and are believed to cause the breakdown of the Rosenbluth separation used to extract form factors and thereby the proton charge radius from electron scattering data.

\section{Prior results}

The accepted value of the proton radius comes from the CODATA 2010 analysis: $r_{p}=0.8775 \pm$ $0.0051 \mathrm{fm}$. This analysis is a compilation of radius results from hydrogen spectroscopy data and electron scattering including the best electron scattering data from MAMI by Bernauer et al. [2]. The data agree with the recent JLab polarized measurement [3]. There have also been several reanalyses and reviews which agree with the CODATA results. In particular, the review by Pohl et al. [4] gives a good summary of the situation.

The muonic hydrogen measurements $[1,5]$ were made as the knowledge of the proton radius limits precision in many electromagnetic physics measurements. The atomic excitation spectrum is perturbed by the finite size of the proton. If one replaces the electron in hydrogen with a muon, due to the greater mass of the muon, the atom becomes much smaller and therefore the muon spends much more time on average inside the proton. This makes muonic hydrogen vastly more sensitive to proton size effects and should lead to a very precise measurement of the proton radius. The measurement by Pohl et al. of $r_{p}=0.84184 \pm 0.00067 \mathrm{fm}$ [1] was highly unexpected and the result was confirmed in 2013 by the analysis of Antognini et al., whose value for the proton electric radius of $r_{p}=0.84087 \pm 0.00039 \mathrm{fm}$ confirmed the existence of the puzzle.

There were initially many suggestions as to what could cause this discrepancy in radius measurements, but none of the suggested solutions to date have been generally accepted as resolving the puzzle. Remaining possible explainations include possible physics beyond the Standard Model (SM), whereby the electron is measuring an electromagnetic radius, while the muon is measuring an electromagnetic radius convoluted with beyond SM effects. There are also suggestions that there might be a currently unconstrained correction in the proton polarizability. Neither is universally accepted but neither has been conclusively discredited.

In order to make progress, it is universally accepted that new data are needed and so there are further low $Q^{2}$ electron scattering experiments planned at JLab and MAMI. The CREMA collaboration will measure the radii of several light nuclei using muonic deuterium and muonic ${ }^{3 / 4} \mathrm{He}$. The MUSE experiment is planned at PSI to measure simultaneous elastic electron and muon scattering from hydrogen. The MUSE experiment will also measure both charge states and can thereby also access twophoton exchange effects, postulated to be responsible for the breakdown of the Rosenbluth separation and which would be enhanced by the enhanced proton polarizability proposition.

In the 1970's and 80's, there were several comparisons of electron and muon scattering [6, 7] which supported universality of the interaction at the $10 \%$ level, but none were precise enough to reveal proton radius issues. The radius of ${ }^{12} \mathrm{C}$ was determined in electron scattering and in muonic carbon atoms and the results agreed within the error bars of the measurements at that time. However, newer analyses of the data may indicate that there is some discrepancy after all.

\section{The MUSE experiment}

The MUSE experiment (see Fig. 1) will take place at the $\pi \mathrm{M} 1$ beam line of the Paul Scherrer Institute (PSI). The $\pi \mathrm{M} 1$ beam line is a secondary beam line and the beam consists of a mixture of pions, electrons and muons with a $50 \mathrm{MHz}$ time structure. We will measure at three different beam momenta: $p_{\text {in }} \simeq 115$, 

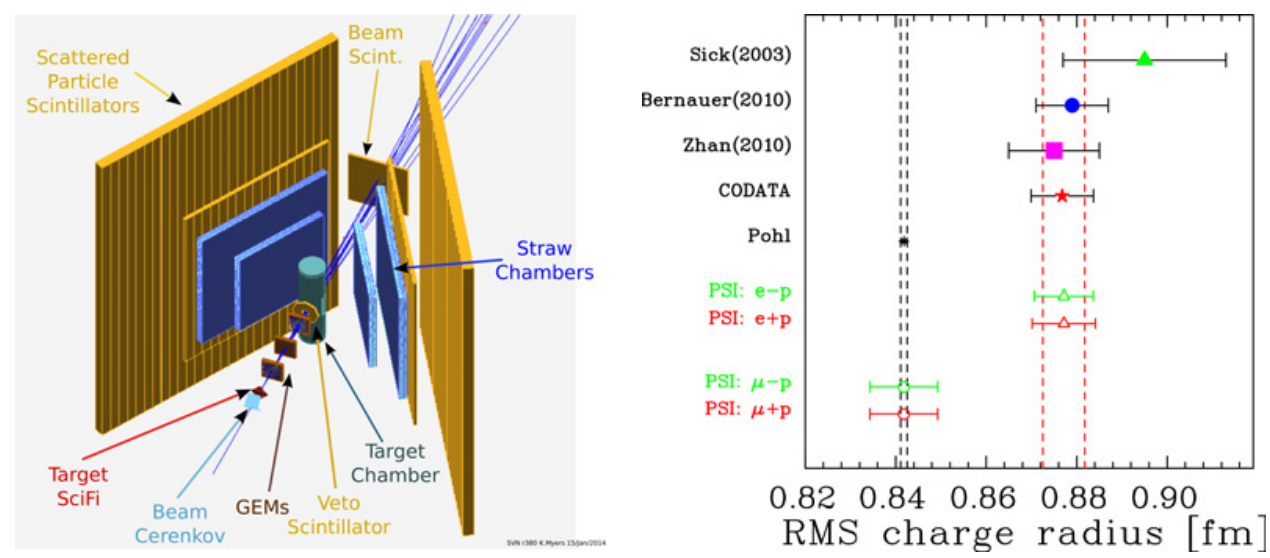

Figure 1. Left: planned MUSE experimental setup. Right: recent extractions of the proton radius from electron and muon based measurements, along with the projected relative uncertainties from the proposed measurements.

153 and $210 \mathrm{MeV} / \mathrm{c}^{2}$. These momenta are chosen to allow good seperation of the different particle types in time and give overlapping kinematic points for good systematic control while providing data in the range of kinematic interest for measurements sensitive to the proton radius.

Due to the large dispersion and the mixed particle nature of the beam, it is necessary to monitor beam timing and angle accurately. This will be achieved by measuring particle timing in two sapphire Cerenkov detectors, following the idea prototyped by Albrow et al. [8] to provide accurate timing of beam particles relative to the beam RF signals and time of flight. These Cerenkov detctors will be located at the intermediate focal point, where the beam enters into the experimental area, and in the target region.

The position and tracking of beam particles will then be achieved using a scintillating fiber ( $\mathrm{SciFi}$ ) detector in the target area, consisting of three layers of forty fibres each, with double-ended maPMT readout. This is followed by a stack of three Gas Electron Multiplier (GEM) chambers taken from the OLYMPUS at DESY [9]. While all the other equipment will be custom made for the experiment, the GEM chambers already exist, are already present in PSI and have been used for test beam times. The SciFi and GEM chambers will be used in the offline analysis to determine the exact angle of the particles entering the target to enable better scattering angle definition.

In order to compensate for the low beam flux of the $\pi \mathrm{M} 1$ beam line, the detector system employed by the MUSE experiment will take the form of a non-magnetic, large-acceptance tracking detector. There will be two identical sectors, one on each side of the beamline. Each side will consist of two ten-layer straw tube tracking chambers (STTs) followed by two layers of fast scintillators for triggering and timing.

The STTs will be based on the PANDA design and will be read out for timing only using PADIWA discriminators and TRB3 boards which provide timing, scalars and trigger logic [10]. The scintillation counter signals will be sent into PADIWA discriminators and TRB3 boards for timing etc., but also into QDCs in order to allow for offline pulse-height timing corrections.

The experimental trigger will be based on the requirement of a particle firing both IFP and target Cerenkov detectors, the SciFi detector and a scattered particle with appropriate timing and position reaching the scattered particle scintillators. The trigger algorithm will be processed in the TRB3 boards used to provide timing and scalar information for each chanel, with one TRB3 trigger master which will make the final trigger decision and distribute the trigger and trigger number to the TRB3s and non-TRB3 parts of the detector system. 
In order to suppress backgrounds caused by muon decays in-flight, there will be an annular veto detector, comprising of eight scintillators, surrounding the entrance to the target scattering chamber. The signals from these detectors will be incorporated in the trigger system as a veto. In order to monitor the beam and ensure stability throughout the experiment, there will be six beam-monitor scintillators with double-ended readout which will be used to monitor beam flux and position.

It is expected that MUSE will produce cross section measurements for the elastic scattering of $\mu^{+/-}$ and $e^{+/-}$with sub-1\% relative precision over a $Q^{2}$ range from 0.002 to $0.07 \mathrm{GeV}^{2}$. Using these data, form factors will be extracted and the resulting radii from muon and electron scattering, and from both charge states, will be compared (see Fig. 1).

\section{Current status and future plans}

The experiment was given conditional approval by the PSI Users' Meeting in January 2013. Since then, there have been several MUSE test beam times during which we have demonstrated and improved upon our understanding of the beam quality, constructed a test readout using MIDAS readout software and investigated the use of several components of our final detector and DAQ systems. We have also incorporated the existing GEM chambers in our MIDAS readout.

In the latter half of 2013, we submitted a funding proposal to NSF for consideration of joint DOENSF funding. The proposal is currently under review and, should it be successful, we plan to begin building the experimental setup in summer 2014, for a "dress rehersal" with half of the detector in late 2015, with the experiment itself taking place for two six-month measurements in 2016 and 2017.

The MUSE experiment is expected to provide crucial missing information in the quest to solve the proton radius puzzle. The simultaneous measurement of electron and muon scattering has recieved physics appoval and the collaboration is in the process of arranging funding for this important experiment.

\section{References}

[1] R. Pohl, A. Antognini, F. Nez, F.D. Amaro, F. Biraben et al., Nature 466, 213 (2010)

[2] J. Bernauer et al. (A1 Collaboration), Phys. Rev. Lett. 105, 242001 (2010), [arXiv: [nucl-ex] 1007.5076]

[3] X. Zhan et al., Phys. Lett. B 705, 59 (2011), [arXiv: [nucl-ex] 1102.0318]

[4] R. Pohl, R. Gilman, G.A. Miller, K. Pachucki, Ann. Rev. Nucl. Part. Sci. 63, 175 (2013), [arXiv: [atom-ph] 1301.0905]

[5] A. Antognini et al., Science 339, 417 (2013)

[6] L. Camilleri, J. Christenson, M. Kramer, L. Lederman, Y. Nagashima et al., Phys. Rev. Lett. 23, 153 (1969)

[7] R. Ellsworth et al., Phys. Rev. 165, 1449 (1968)

[8] M. Albrow, H. Kim, S. Los, E. Ramberg, A. Ronzhin et al., JINST 7, P10027 (2012), [arXiv: [ins-det] 1207.7248]

[9] R. Milner et al., Nuclear Inst. and Methods in Physics Research A 741, 1 (2014), [arXiv: [ins-det] 1312.1730]

[10] TRB homepage containing full specification and documentation: trb.gsi.de 